\title{
Impacts of Silver Nanoparticle Ingestion on Pigmentation and Developmental Progression in Drosophila
}

\author{
S. Catherine Silver Key', Denise Reaves', Fran Turner' ${ }^{1}$, John J. Bang ${ }^{2 *}$
}

${ }^{1}$ Department of Biology, North Carolina Central University, Durham, NC 27707, USA; ${ }^{2}$ Departments of Environmental, Earth, and Geospatial Science, North Carolina Central, University, Durham, NC 27707, USA

Received: February 12, 2011 / Accepted: May 14, 2011

\begin{abstract}
In recent years, the advent of nanomaterial use has increased exposure rates and raised health concerns. However, the toxicology profiles of many nanomaterials are far from complete for various reasons. In this study, Drosophila melanogaster, commonly called fruit flies, were exposed to one of the most widely used nanomaterials, silver nanopowder (Ag NP), to assess its toxicity and determine if $D$. melanogaster would be a good model organism for nanotoxicology studies. Comparison of developmental progression amongst groups of flies ingesting different Ag NP concentrations (0.05\%/ 90 ppm-5.0\%/ 9000 ppm), revealed that hatch rates were unaffected, but that larval progression was impeded at any dosage of Ag NP. At $0.3 \%$ Ag NP an approximate LD50 was observed. Additionally, a distinctive phenotype was observed among emergent adults (F1 generation) that arose from larvae exposed to Ag NP which included reduced body pigmentation accompanied by shortened life span and abnormal climbing behavior. The phenotype prompted speculation that Ag NPs may affect the dopamine and/or the stress response pathway(s).
\end{abstract}

Keywords: Silver nanoparticles, exposure, development, pigmentation, Drosophila.

Abbreviations and Acronyms: $\mathrm{Ag}=$ Silver, $\mathrm{NP}=$ nanoparticle or nanopowder, $\mathrm{P}=$ parental generation, $\mathrm{Fl}=$ adult flies of the first filial generation, L=larvae of Fl generation.

\section{Introduction}

Since the discovery of fullerene and carbon nanotubes, the use of engineered nanomaterials has exponentially increased for various applications. Among the nanomaterials used, silver $(\mathrm{Ag})$ in the form of nanoparticle and nanopowder (NP) is one of the most popular for various applications including antibacterial uses (Sharma et al., 2009). In fact, the use of silver for therapeutic and industrial purposes traces back to the time period long before nanotechnology (Chen and Schluesener, 2008). Today, because Ag NPs have well-documented beneficial effects due to their unique physicochemical properties that larger counterparts do not carry, ultrafine and nanopowder forms of silver penetrate tissues more effectively. Thus, Ag NP is not only used in burn and diabetic ulcer creams, but is also used in common household items such as first aid bandages, cosmetics, appliances, and clothing (Liu et al., 2010; Kumari et al., 2010). These products are presumed safe once in consumer hands. However, industrial workers, physicians, and researchers are increasingly exposed to a plethora of nanoparticles, the impact of which is currently unknown. While the intended uses of these materials are to benefit human health, comprehensive toxicological profiles for nano materials are yet to come.

The toxicity of silver nanoparticles (Ag NP) has been documented in a large number of in vitro studies using a number of cell lines including but not limited to human hepatoma cells (Kim et al., 2009), PC1 2 neuronal cells (Powers et al., 2010), BRL3A rat liver cells, germline stem cells (Braydich-Stolle et al., 2005), human lung fibroblasts, and gliablastoma cells (AshaRani et al., 
2009). However, a fewer number of in vivo studies using animal models has been conducted (Choi et al., 2009; Ahamed et al., 2010b; Posgai et al., 2009; Kumari et al., 2009; Li et al., 2010; Wu et al., 2009). The results from previously conducted studies provided consistent data indicating that Ag NP exposure elicits both oxidative stress and apoptotic responses, and that the particles can accumulate in organs including the brain.

Drosophila melanogaster, commonly known as the 'fruit fly', is an excellent organism for answering many questions regarding human health because Drosophila shares some $60-70 \%$ homology to human genes including those that encode molecules essential for carcinogenesis, pigmentation, and the nervous system (Rubin, 2000; Rand, 2010). Because of a well-established genetics, short life cycle (10-14 days from embryo to adulthood), and highly conserved genes, many developmental discoveries have been made in Drosophila that apply to humans. In addition, the average cost for conducting an in vivo exposure experiment by using Drosophila is a lot lower than that of other animal models. For these reasons, this study aims to assess the feasibility of using Drosophila as an in vivo model for future exposure-induced toxicity experiments on engineered nanomaterials including $\mathrm{Ag}$ NP.

The three major entry modes of nanoparticles or nanomaterials typically include inhalation, epidermal absorption, and ingestion. Currently, Ag NP is used in food packaging as well as in medical treatment procedures (Johnston et al., 2010). Ag NP also has been shown to accrue in waste, and the silver particulates could contaminate consumer's food and drinking water (Fernandez et al., 2010; Erickson, 2009; Kim et al., 2010). Further, Ag NPs have been shown to accumulate in animal and human tissues and organs (Jonas et al., 2007; Larese et al., 2009; Ahamed et al., 2010; Tang et al., 2010a). Given the great potential for human ingestion of Ag NP, this study aims to understand the impacts of Ag NP exposure through the ingestion mode on phenotypic outcomes and behavioral characteristics of Drosophila. The implications for Ag NP effect on pigmentation, life span, and behavioral response are discussed.

\section{Materials and Methods}

\section{Experimental Design}

Silver nanoparticles (Ag NP) of different concentrations ranging between $0.05 \%$ (89.3 ppm) and 5.0\% (8930 ppm) were prepared to determine the exposure effects at different developmental stages. A manageable number of newly emerged parental flies were either exposed immediately to a given $\mathrm{Ag}$ NP concentration ( $\mathrm{P}^{+}$, exposed parents) or placed in food vials that contained control food ( $\mathrm{P}_{-}$, unexposed parents). The eggs laid by these parents were collected and transferred to either Ag NP-containing food ( $\mathrm{L}^{+}$, larvae exposure) or to food lacking Ag NP ( $L$; larvae unexposed) as described in the Drosophila Nanopowder Exposure section below. Progression through all developmental stages was monitored: egg hatching into the F1 generation of larvae, larval progression, pupal stage development, and eclosion of adult flies. Groups of Fl adult flies were then monitored for the presence or absence of pigment and lon-

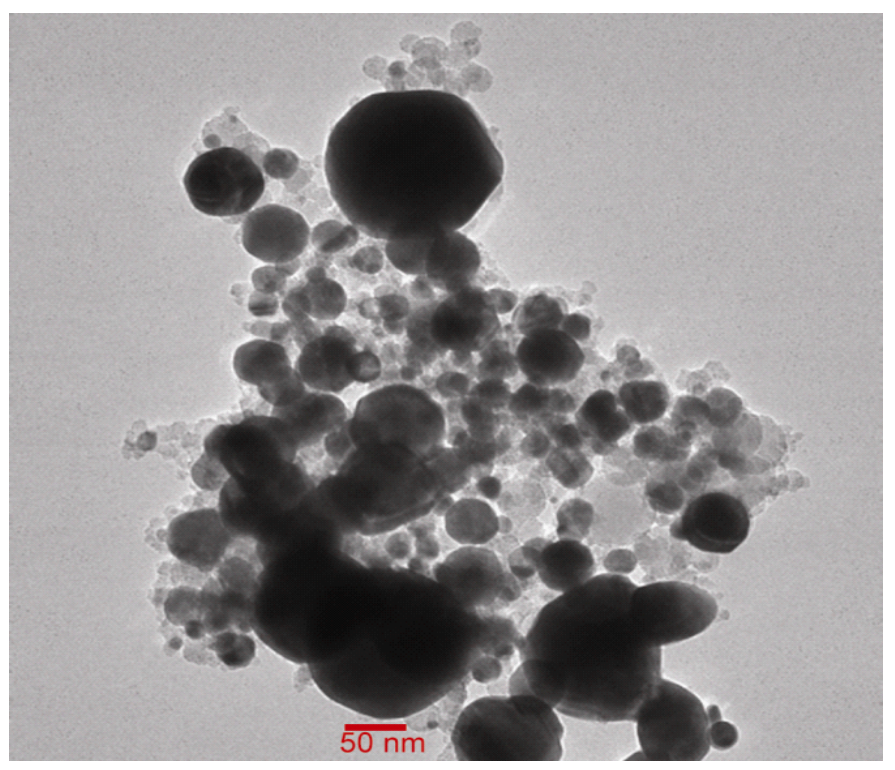

Fig. 1. Ag NP under Transmission Electron Microscopy. The Ag NP suspension was put on a Cu grid for a TEM exam. A smaller number of $\mathrm{Ag}$ NP is bigger than the average values presented by the DLS. Agglomeration of smaller particles occurs during the drying process and there are limitations of the manufacturing process. However, the majority of the particles are within the average values presented by the DLS.

gevity for up to 20 days. Experiments were conducted by using triplicate samples with each set comprised $\sim 200$ eggs. During each developmental stage, observation of overt phenotypes was documented. Fl generation adults that had emerged from larvae exposed to $\mathrm{Ag} \mathrm{NP}\left(\mathrm{L}^{+}\right)$were assessed for climbing behavior as described below. Data from all replicate experiments was assessed using a student T-test shown elsewhere.

\section{Statistical analysis of Developmental Progression, Maternal versus Larval Exposure Analysis, and Life Span Assessment}

All calculations for average, standard deviation, and t-test were performed using Microsoft Excel version 2007 formulas. Triplicate samples were used during the study unless otherwise specified. For the developmental progression analysis in Figure 2 , three replicates of $\sim 200$ eggs per condition were run. As the percent eggs laid was arbitrarily set to $100 \%$, the average percent and standard deviation of eggs that progressed to each developmental stage (larval, pupal, and adult (shown in both Figure 2 and Table 1)) were calculated. T-tests were performed for each stage (larval, pupal, and adult) using the unexposed group as a control. For the analysis on exposure timing effect as shown in Figure $3 b$, five sets of 30 eggs per condition were run. The average and standard deviation were calculated using Microsoft Excel 2007 formulas. A two-tailed T-test was run comparing the following data sets for maternal effect assessment: $\mathrm{P}^{-} / \mathrm{L}^{-}$versus $\mathrm{P}^{+} / \mathrm{L}^{-}$and $\mathrm{P}^{-} / \mathrm{L}^{+}$versus $\mathrm{P}^{+} / \mathrm{L}^{+}$and the following sets for larval effect assessment: $P^{-} / L^{-}$versus $P^{-} / L^{+}$ and $\mathrm{P}^{+} / \mathrm{L}^{-}$versus $\mathrm{P}^{+} / \mathrm{L}^{+}$, where $\mathrm{P}=$ parent, $\mathrm{L}=\mathrm{F} 1$ larval generation, '-' indicates unexposed and '+' indicates Ag NP exposed. 
Table 1. Outcomes of Exposure to Silver Nanopowder on developmental progression, life expectancy, reproductive ability, and pigmentation.

\begin{tabular}{lllll}
\hline $\begin{array}{l}\text { Percent Silver } \\
\text { Nanopowder (Ag NP) }\end{array}$ & $\begin{array}{l}\text { \% Embryos develop } \\
\text { to adults }\end{array}$ & $\begin{array}{l}\text { Survival Time } \\
\text { (in days) }\end{array}$ & $\begin{array}{l}\text { F2 Generation } \\
\text { produced? }\end{array}$ & $\begin{array}{l}\text { Abdominal/Thoracic } \\
\text { Pigmentation }^{3}\end{array}$ \\
\hline 5.0 & $7 \pm 1.8$ & $1 \pm 0.3$ & no & none \\
2.0 & $1 \pm 0.2$ & $1 \pm 0.3$ & no & none \\
1.0 & $1 \pm 0.2$ & $2 \pm 0.2$ & no & none \\
0.5 & $20 \pm 6.0$ & $18 \pm 2.0$ & no & none to very light \\
0.3 & $45 \pm 3.7$ & $19 \pm 2.4$ & yes & light to normal \\
0.1 & $60 \pm 4.6$ & $20 \pm 1.6$ & yes & light to normal \\
0.05 & $45 \pm 6.7$ & $20 \pm 0.8$ & yes & light to normal \\
control & $83 \pm 7.3$ & $20 \pm 1.0$ & yes & normal \\
\hline
\end{tabular}

${ }^{1}$ The standard deviations are shown $( \pm)$. T-tests indicate significant differences in the percent of Ag NP-exposed embryos progressing to adulthood as compared to control: $0.3 \%(p=0.044), 0.5 \%$ ( $p$-value $=0.012), 1.0 \%$ ( $p$-value $=0.003), 2.0 \%(p$-value $=0.003)$ and $5.0 \%$ ( $p$-value $=0.005) .{ }^{2}$ Standard deviations are shown $( \pm)$. T-test calculations indicate that survival time differences for $5.0 \%$ ( $p$-valve $=0.0004), 2.0 \%(p$-value $=0.0006)$ and $1.0 \%(p$-value $=0.0011)$ were significant compared to control. Survival length in days was not significantly different for all other concentrations. Adults were observed for a 20 -day period. ${ }^{3}$ Adult pigmentation was determined at the day of death. Emergent Fl generation adult flies were maintained on a continuous concentration of silver nanoparticle-containing food during parental and larval stages $\left(\mathrm{P}^{+} / \mathrm{L}^{+}\right.$condition). $\mathrm{Fl}$ generation adults were observed every 2-3 days for up to twenty days.

For the life span analysis, replicate runs of at least 50 flies per conditions were observed for 20 days after eclosion from pupal cases. Because flies in each group died at different times during each run the weighted average was calculated to assess approximate length of survival for the group as a whole. Statistical comparisons between the control group and each Ag NP condition were assessed using a one-tailed T-test.

\section{Silver Nanopowder (Ag NP)}

The TEM image of the silver nanopowder (Ag NP) used in this ingestion exposure study is shown in Figure 1. Commercially available Ag NP was used (Sun Innovations Corp., Fremont, CA). Ag NP was added to filtered water (NanoPure Diamond from Barnstead). A suspension of Ag NP in purified water was prepared at different concentrations so that their converted final concentration after mixing with food would be equivalent to the concentration levels used for the study (i.e., 0.05, 0.1, 0.3, 0.5, $1.0,2.0$, and $5.0 \%$ in dry Ag NP to dry food weight). For example, for $0.5 \%$ Ag NP food (dry weight of Ag NP in dry food weight), $125 \mathrm{mg}$ of Ag NP was mixed in $140 \mathrm{ml}$ of water. This makes up a $893 \mathrm{ppm}$ concentration of Ag NP in prepared food which is equivalent to $8.3 \mathrm{mM} \mathrm{Ag} \mathrm{NP}(125 \mathrm{mg} / 108 \mathrm{mg} /$ mole/0.14L of water). Before Ag NP was mixed with the fly food, the Ag NP suspension was sonicated for at least $30 \mathrm{~min}$ utes to obtain a homogeneous dispersion, and the physical dimensions of the Ag NP in suspension were evaluated by using a dynamic light scatter (DLS using a CGS 3 spectrometer (ALV
$\mathrm{GmbH}$, Germany) equipped with a helium-neon laser (633.4 $\mathrm{nm}$ ) and goniometer). The average radius of the Ag NP used was $16.7 \pm 0.30 \mathrm{~nm}$. The average width was $11.3 \pm 0.30 \mathrm{~nm}$. DLS measures the hydrodynamic diameter particles in suspension and presents the average values of the measured particles.

\section{Drosophila Nanoparticle Exposure}

A commonly used control fly strain, w1118 (Bloomington Stock \# 3605), was chosen to initiate nanoparticle exposure experiments. Fifty virgin female and 30 male flies were collected and placed (within 24 hours) into cages capped with grape juice agar plates for egg collection. Each plate contained 1 gram of Nutri-FlyTM BF food (catalog\# 66-1 12, Genesee Scientific) with a different concentration of silver nanoparticles $(0.05 \%$, $0.1 \%, 0.3 \%, 0.5 \%$ and $1.0 \%$ ) or control food ( $0 \%$ Ag NP). Four different exposure settings were created. 1) Newly emerged parental flies (P generation) were exposed to Ag NP, their eggs collected and transferred to hatch in food containing Ag NP for the larval (L) exposure $\left(\mathrm{P}^{+} / \mathrm{L}^{+}\right)$. 2) Newly emerged $\mathrm{P}$ generation flies were exposed to Ag NP and the larvae were unexposed $\left(\mathrm{P}^{+} / \mathrm{L}^{-}\right)$. 3) Exposure-free $\mathrm{P}$ generation flies and their progeny larvae exposed to Ag NP $\left(\mathrm{P}^{-} / \mathrm{L}^{+}\right)$, and 4) Exposure-free $\mathrm{P}$ generation flies and then progeny larvae were also unexposed $(P-$ (L)). Forty-eight hours after flies were transferred to food, eggs were collected for 24 hours at $25^{\circ} \mathrm{C}$. Because w 1118 lacks eye pigmentation, the Oregon RS strain (Bloomington Stock \#4269) with wildtype eye color was used to look at the impacts of $\mathrm{Ag}$ 

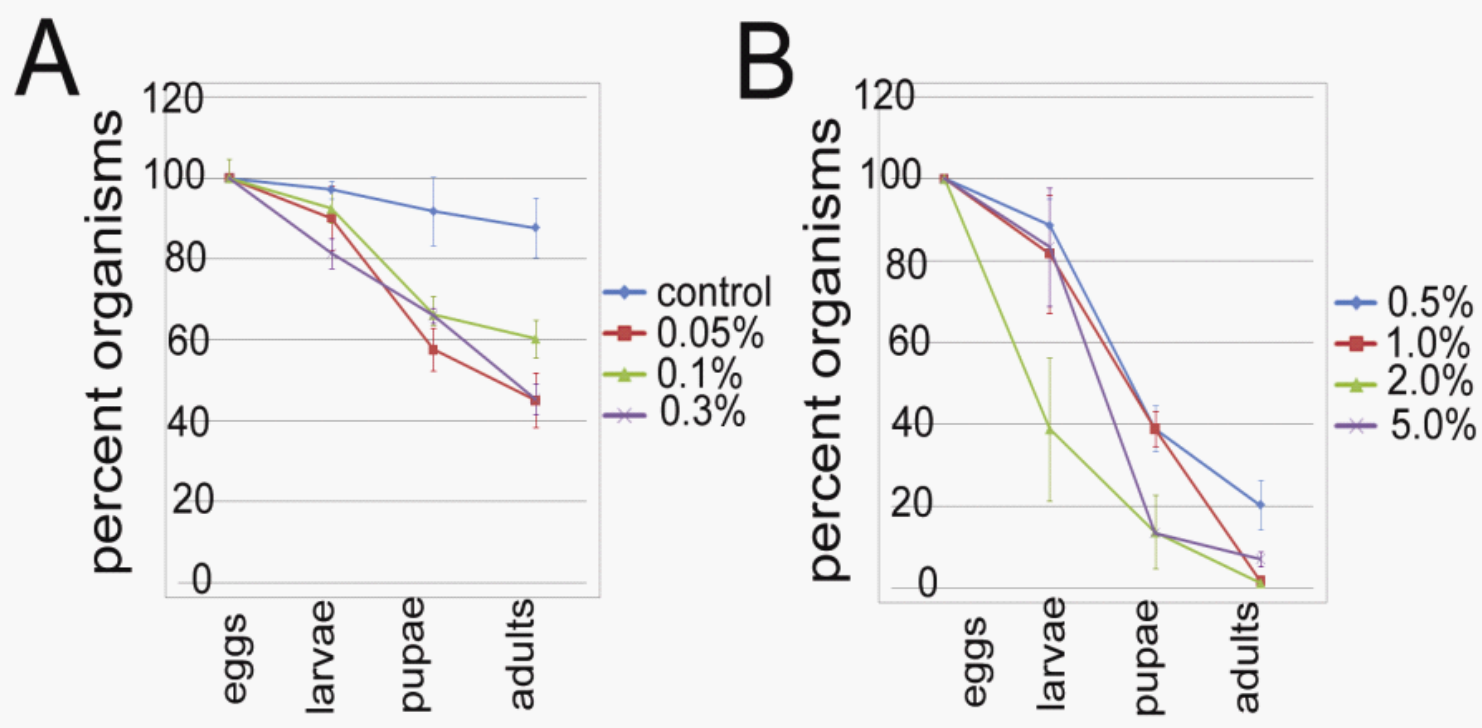

Fig. 2. Impacts on Developmental Progression by Silver Nanopowder (Ag NP) Ingestion. Newly emerging P generation flies were fed on Ag NP containing food; subsequently, their larvae were exposed to Ag NP-containing food $\left(\mathrm{P}^{+} / \mathrm{L}^{+}\right.$condition) and assessed for progression through larval, pupal and adulthood ( $F 1$ generation). In Panels $A$ and B, results are shown as percentages of live organisms at each developmental stage (egg, larvae, pupae, or $\mathrm{Fl}$ adult) in the form of dose response curves for each concentration of Ag NP as indicated. Panel A, non-exposed, control fed organisms (( $\left.\mathrm{P}^{-} / \mathrm{L}^{-}\right)$, blue diamonds), $0.05 \% \mathrm{Ag} \mathrm{NP}$ (red squares), $0.1 \% \mathrm{Ag} N \mathrm{P}$ (green triangles), and $0.3 \% \mathrm{Ag} \mathrm{NP}$ (purple Xs). Panel B, results from different Ag NP concentration exposures; $0.5 \%$ Ag NP (blue diamonds), $1.0 \% \mathrm{Ag} \mathrm{NP}$ (red squares), $2.0 \% \mathrm{Ag} \mathrm{NP}$ (green triangles) and 5.0\% Ag NP (purple Xs) are directly comparable to Panel A. A total of three replicate experiments were run using approximately 200 eggs per experimental condition. Since the number of eggs laid per condition was equivalent, the percent of eggs laid was arbitrarily set at $100 \%$ for each condition and the standard deviation (bars) is shown for larval, pupal, and adult stages. One-tailed T-test analysis was completed by comparing percent larvae, pupae, or adult flies in each Ag NP condition to the percent of larvae, pupae, or adult flies surviving in the control condition. For $0.5 \%$ (p-value $=0.033), 1.0 \%$ (p-value $=0.028), 2.0 \%$ ( $p$-value $=0.009)$ and $5.0 \%(0.009)$ Ag NP exposure, the percent of pupal cases observed was significantly fewer than in the control condition. Further, the percent of adult eclosion was also significantly lower among the $\mathrm{Fl}$ generation flies exposed to higher $0.3 \% \mathrm{Ag}$ NP concentrations and higher.

NP exposure $(0.05 \%$ and $1.0 \%)$ on eye color.

All stages were kept at $25^{\circ} \mathrm{C}$. All experiments as describe above $\left(\mathrm{P}^{+} / \mathrm{L}^{+}, \mathrm{P}^{+} / \mathrm{L}^{-}, \mathrm{P}^{+} / \mathrm{L}^{-}\right.$and $\left.\mathrm{P}^{-} / \mathrm{L}^{-}\right)$were completed in triplicate. Collected eggs on plates containing the fly food with each concentration of silver $(\mathrm{Ag})$ nanoparticle powder were counted immediately and then 24 hours later to determine the hatch rate. The numbers of unhatched eggs, larvae, pupae, and adults were counted and recorded. All data was entered into Excel spreadsheets, with averages, and standard deviations calculated using Excel formulas (Microsoft Office Excel 2007). For the longevity study, the filial generation adult flies (i.e. F1), which resulted from larvae fed $\mathrm{Ag} N \mathrm{~N}$, were maintained on food that either had Ag NP or lacked Ag NP for approximately 3 weeks and observations recorded in table format.

\section{Assessment of Pigmentation}

Emergent flies ( $F 1$ generation) from all four exposure groups $\left(\mathrm{P}^{+} / \mathrm{L}^{+}, \mathrm{P}^{+} / \mathrm{L}^{-}, \mathrm{P}^{-} / \mathrm{L}^{+}, \mathrm{P}^{-} / \mathrm{L}^{-}\right)$were evaluated for pigmentation by using a Leica LZ6 steromicroscope. Flies were photographed using a FinePix F2O SE digital camera and XD-picture card (Fuji
Photo Film, Ltd. Tokyo, Japan) temporarily mounted to the scope using the Orion SteadyPixTM Deluxe stand (Orion Telescopes \& Binoculars, Watsonville, CA). Three independent experiments were completed.

\section{Climbing Behavior Analysis}

Climbing assays were performed on $\mathrm{F} 1$ generation flies as previously described (Jimenez-Del-Rio et al., 2010; Chaudhuri et al., 2007) with the exceptions that the height of the climb was $7 \mathrm{~cm}$ and the age of the flies in this study was specified at 9-days old. The flies (F1 generation) emerging from both groups of larvae, either Ag NP exposed or non-exposed, were tapped down to induce climbing behavior and video recorded (Sharp Viewcam Liquid Display Camcorder model VL-E760U). Video recordings were transferred to digital format using a DVD Maker USB 2.0 (USB2800D, KWorld (USA) Computer Co., Ltd, Irvine, CA). Windows Movie Maker software was used to generate snapshots for Figure $6 B$ and to create the video in supplementary materials. Video play-back was halted using the 'still' function to measure the rate of climbing for the first 5 
A
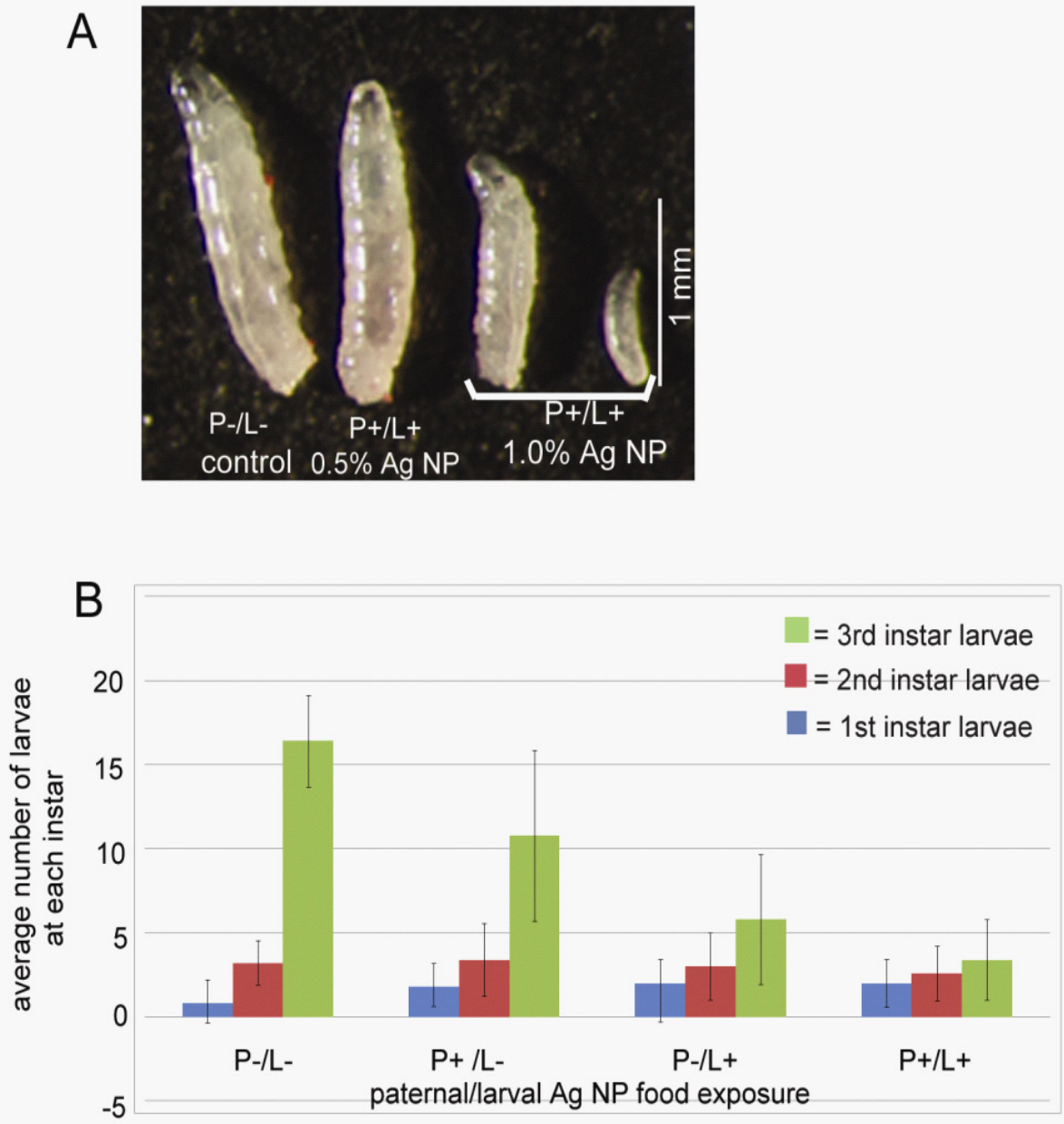

Fig. 3. Stage Dependent Effects of Ag NP Exposure on Larval Progression. Five plates of 30 eggs were collected from flies consuming a diet consisting of either $1.0 \% \mathrm{Ag} \mathrm{NP}$ or control food (no exposure) on GJA plates. Eggs were transferred to GJA plates containing either control or $1.0 \% \mathrm{Ag} N \mathrm{~N}$ food. In both panels $A$ and $\mathrm{B}_{,}\left(\mathrm{P}^{-} / \mathrm{L}^{-}\right)$group represents no exposure for parents or larvae; $\left(\mathrm{P}^{+} / \mathrm{L}^{-}\right)$ represents exposed parents with non-exposed larvae; $\left(\mathrm{P}^{-} / \mathrm{L}^{+}\right)$indicates non-exposed parents with exposed larvae; $\left(\mathrm{P}^{+} / \mathrm{L}^{+}\right)$indicates both parents and larvae were exposed. Panel A. Image of larval size in $\mathrm{P}^{-} / \mathrm{L}^{-}$experimental condition versus two concentrations of $\mathrm{Ag}$ NP: $0.05 \%$ and $1.0 \%$. Panel B. Graph indicating the average number of larval progressing to each length: 1 st instar size $0.5<1 \mathrm{~mm}$ (blue bars) 2nd instar size (red bars) $=1<1.5 \mathrm{~mm}$, and 3rd instar (green bars) $=1.5-2 \mathrm{~mm}$ in length. Standard deviation are shown (thin black bars). Two-tailed T-test comparisons between parental exposure and non-exposure groups indicated the difference was not significant with $p$-values greater than $0.05\left(\mathrm{P}^{-} / \mathrm{L}^{+}\right.$versus $\mathrm{P}^{+} / \mathrm{L}^{+}$and $\mathrm{P}^{+} / \mathrm{L}^{-}$versus $\left.\mathrm{P}^{+} / \mathrm{L}^{+}\right)$. Comparison of larval exposure groups indicated a significant difference for the $3^{\text {rd }}$ instar stage only for the following groups: $\mathrm{P}^{+} / \mathrm{L}^{-}$group versus the $\mathrm{P}^{+} / \mathrm{L}^{+}$group $(p$-value $=0.008$ ) and $\mathrm{P}^{-} / \mathrm{L}^{-}$versus $\mathrm{P}^{-} / \mathrm{L}^{+}$group $(\mathrm{p}$-value $=0.007)$.

flies that ascended to the top (6-7 $\mathrm{cm}$ for control flies) and the time recorded. Locomotion speed was calculated by dividing the distance by the time elapsed. Trials were run three times for each concentration from $0.05 \%(89.3 \mathrm{ppm})$ to $0.5 \%$ (893 ppm) of Ag NP.

\section{Statistical Analysis on Climbing Behavior}

Statistical analysis on climbing behavior data was accomplished using Microsoft Excel 2007 two-tailed T-test for 15 independent flies per condition.

\section{Results}

\section{Impairment of Developmental Progression by Ingested Silver Nanoparticles}

To determine the developmental stage(s) at which fruit flies may be most sensitive to dietary silver nanoparticles (Ag NP) exposure, Drosophila melanogaster adult flies and their progeny were exposed to non-coated Ag NP and monitored throughout development from egg hatching to adult ecolosion. Newly emerged, unexposed male and female parental flies ( $P$ generation) were collected and immediately exposed to food with $\mathrm{Ag}$ 


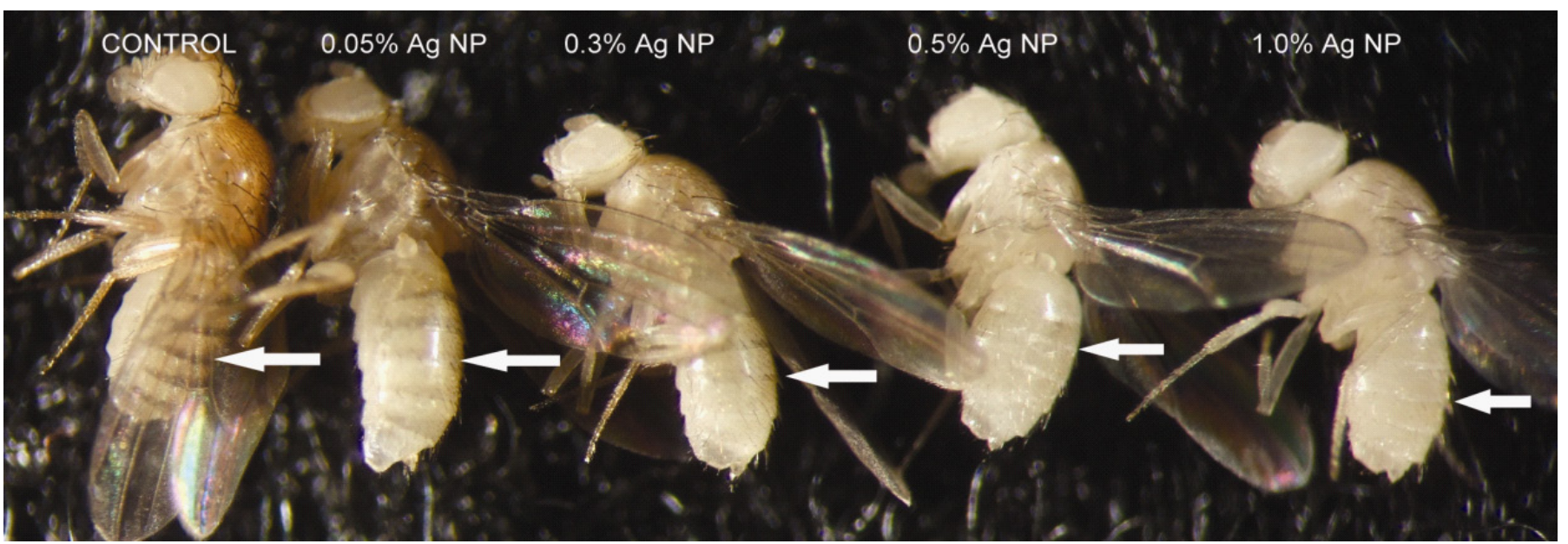

Fig. 4. Inverse Correlation between the level of Melanization and Exposure Level of Ag NP. F1 generation w1 118 flies emerging from pupal cases resulting from parental $(P)$ and larval $(L)$ exposure to a continuous diet of control (no exposure $\left(\mathrm{P}^{-} / \mathrm{L}^{-}\right)$) or silver nanoparticles $\left(A g N \mathrm{P}_{1}\left(\mathrm{P}^{+} / \mathrm{L}^{+}\right)\right.$). White arrows point to the third abdominal stripe in each female adult. Control, $0.05 \% \mathrm{Ag}, 0.3 \% \mathrm{Ag}$ and $0.5 \% \mathrm{Ag}$ flies were less than 8 hours old, while the $1.0 \%$ Ag NP fly was 24 hours old.

$\mathrm{NP}\left(\mathrm{P}^{+}\right)$or a control food without Ag NP ( $\left.\mathrm{P}^{-}\right)$. The number of eggs laid by the parental $(P)$ adult flies with a history of exposure of any amount of Ag NP during their adult stage (i.e., $\mathrm{P}^{+}$) was comparable to the number of eggs laid by the flies fed non-exposed food (P-) ( 200 \pm 50 per overnight plate; $p$-value $=0.05$ for all concentrations versus unexposed control). In addition, the hatch rates of eggs laid by both $\mathrm{P}^{+}$mothers (at $1.0 \% \mathrm{Ag} \mathrm{NP}$ exposure) and $P$ - mothers were also comparable at nearly $95 \pm 5 \%(p-$ value $=0.14)$. However, when the Fl generation larvae were exposed to any amount of Ag NP, developmental progression of the larvae to the pupal stage presented a different picture. In the group of larvae exposed to Ag NP at concentrations of $1.0 \%$ and above, only a small percentage of the F1 generation embryos progressed through the larval stages to reach the pupal stage ( $13-40 \%$, Figure $2 \mathrm{~B})$ while around $92 \pm 8 \%$ of non-exposed larvae reached the pupal stage (Figure 2A). Compared to the non-exposed group in which $87 \pm 7 \%$ of the $\mathrm{F} 1$ generation embryos progressed to adulthood, the eclosion rates of larvae exposed to higher Ag NP concentrations are dramatically reduced to $1-7 \%(1.0 \%$ and above, Figure $2 \mathrm{~B}, \mathrm{p}$-values less than 0.05: i.e., $0.003,0.003$ and 0.005 respectively). At the $0.5 \%$ Ag NP concentration (893 ppm), about $39 \pm 6 \%$ of embryos progressed to the pupal stage with only $20 \pm 6 \%$ emerging as adults (Figure $2 \mathrm{~B}, \mathrm{p}$-value $=0.030$ ). For larvae fed less than $0.3 \% \mathrm{Ag} \mathrm{NP}$, eclosion rates appeared to plateau at $65 \pm 2 \%$ and never achieved an eclosion rate equivalent to control fed $\mathrm{F} 1$ generation flies $(87 \pm 7 \%)$ under our experimental conditions (Figure 2A, compare $0.1 \%$ and control). When parental flies and their larvae $(\mathrm{L})$ were exposed to a diet containing $0.3 \% \mathrm{Ag}$ $\mathrm{NP}\left(\mathrm{P}^{+} / \mathrm{L}^{+}\right)$, then more than $65 \%$ of eggs developed to the pupal stage with $45 \pm 4 \%$ eclosing as adults (compare $2 \mathrm{~A}$ to $2 \mathrm{~B}$ ). Thus, with about half the embryos eclosing as adults, the $0.3 \% \mathrm{Ag}$ NP conditions approximated a lethal dose 50 (LD50).

It was apparent that $\mathrm{Fl}$ generation larvae from the two groups differed in size (Fig 3A). While the non-exposed larvae grew to a size of $\sim 2 \mathrm{~mm}$, the majority of the $1.0 \% \mathrm{Ag} \mathrm{NP}$ fed larvae remained at a length of $1 \mathrm{~mm}$ or less over the same 5 -day period (Figure 3A). In order to determine if timing of exposure to Ag NP is a critical factor for developmental progression, a combination of four exposure conditions was tested as shown in Figure $3 B$ (i.e., $\mathrm{P}^{+} / \mathrm{L}^{+}$(parent exposed/larvae exposed), $\mathrm{P}^{+} / \mathrm{L}^{-}$(parent exposed/larvae non-exposed), $\mathrm{P}^{-} / \mathrm{L}^{+}$(parent non-exposed/larvae exposed), $\mathrm{P}^{-} / \mathrm{L}^{-}$(parent non-exposed/ larvae non-exposed)).

When the $\mathrm{Fl}$ generation of larvae were not exposed to $\mathrm{Ag}$ $\mathrm{NP}$, the parental (P) exposure history, either $\mathrm{P}^{+}$or $\mathrm{P}^{-}$, did not influence the numbers of larvae reaching the 3rd instar stage as much as the other cases where the larval history of exposure, either $\mathrm{L}^{+}$or $\mathrm{L}^{-}$, was compared (i.e. $\mathrm{P}^{+} / \mathrm{L}^{+}$vs. $\mathrm{P}^{+} / \mathrm{L}^{-}$or $\mathrm{P}^{-} / \mathrm{L}^{+}$vs. $\left.\mathrm{P}^{-} / \mathrm{L}^{-}\right)$. When larvae are exposed to Ag NP, noticeably fewer larvae progressed to 3 rd instar, regardless of parental exposure (Figure 3B). Two-tailed T-tests indicate that the effect of Ag NP exposure at the parental stage $(P)$ does not significantly affect larval progression at all three larval stages (i.e. p-value greater than 0.05). Rather, larval progression to the 3rd instar stage appears significantly impacted if exposure happens during their larval stage. For exposed parental groups $\left(\mathrm{P}^{+} / \mathrm{L}^{-} \mathrm{vs}\right.$ $\left.\mathrm{P}^{+} / \mathrm{L}^{+}\right)$, for example, the history of exposure at the larval stage showed a statistically significant difference in reaching the $3^{\text {rd }}$ instar stage ( $p$-value $=0.008$ ). In a similar way, when parents do not have an exposure history, the Ag NP exposure at a larval stage showed a difference at a statistically significant level in progressing to the $3^{\text {rd }}$ instar stage ( $p$-value $=0.007$ ). Thus, it appears that maternal exposure to the Ag NP does not significantly impact larval development. Instead, Ag NP exposure during larval stages seems to negatively affect larval progression.

\section{Exposure Timing and Concentration Dependent Pigmentation Level in Adult Flies}

The few w 1118 adult flies (Fl generation) that emerged at the initial tests of $1.0 \%$ Ag NP appeared to completely lack 


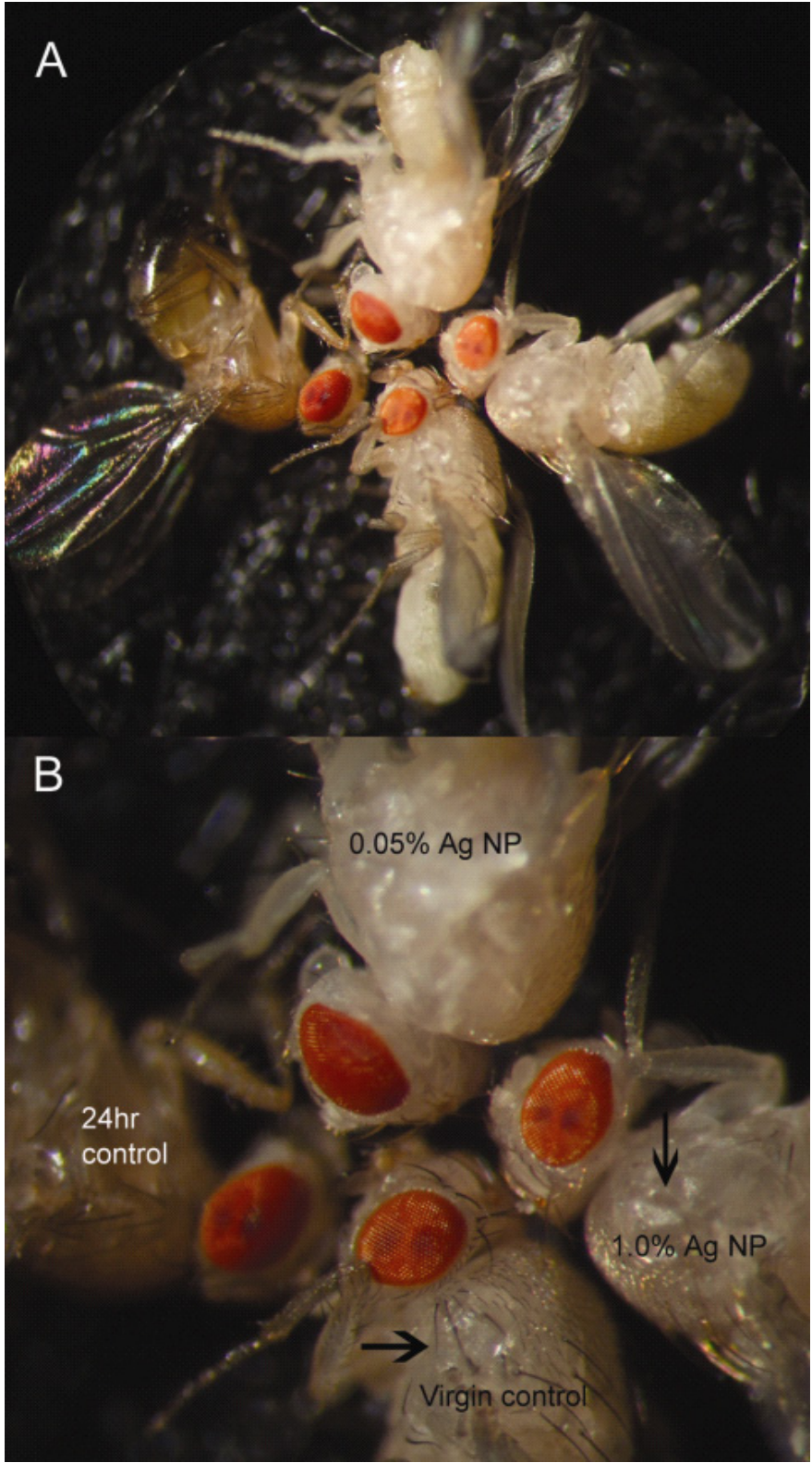

Fig. 5. Impacts of Ag NP exposure targeting on Specific Body Parts sparing Eyes. Images of the Oregon R Drosophila strain Fl generation adults emerging from either a vial with control food $\left(\mathrm{P}^{-} / \mathrm{L}^{-}\right)$or $1.0 \% \mathrm{Ag}$ $\mathrm{NP}$-containing food $\left(\mathrm{P}^{+} / \mathrm{L}^{+}\right)$. Panel A. Male flies emerging from the nonexposed conditions are either $24 \mathrm{hrs}$ old or newly emerged virgin which are naturally less pigmented ( 9 and 6 o'clock positions, respectively), the $0.05 \%$ Ag NP (1 2 o'clock position), or $1.0 \%$ Ag NP (3 o'clock position) in Panels $A$ and $B$ were digitally captured as indicated in Materials and Methods. Panel B. Higher magnification of eyes and bristles (black arrows).

body pigmentation. To investigate whether this phenomenon of loss of pigmentation is a gradual event or if there is a threshold exposure level, experiments were conducted with doses ranging from $0.05 \%$ to $0.5 \%$, retaining $1.0 \%$ as a point of reference. The flies emerging from larvae exposed to a dose of $0.5 \%$ dietary Ag NP, regardless of parental exposure, also lacked detectable body pigmentation. Head, thorax, abdomen, legs, and
A

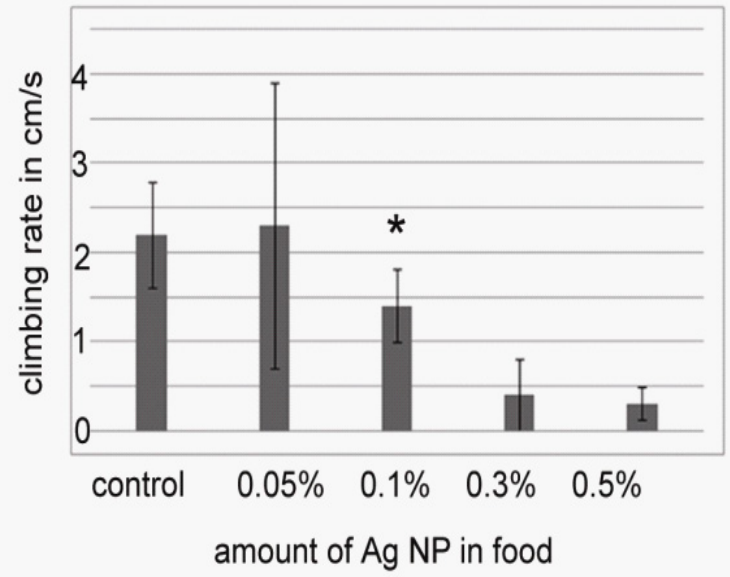

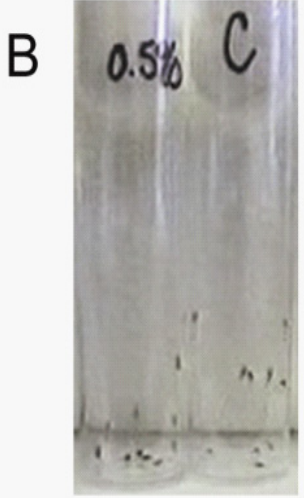

Os

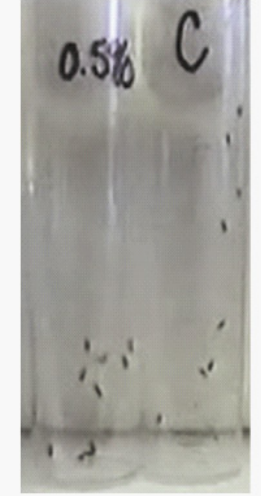

$5 s$

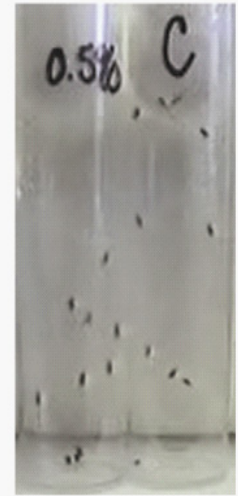

10s
Fig. 6. Impedance in Climbing Behavior after Exposure to Ag NP. Panel A, Climbing behavior was monitored using video analysis of 9-day old w1118 F1 generation adults emerging from continuous developmental exposure to control $\left(\mathrm{P}^{-} / \mathrm{L}^{-}\right)$or Ag NP-containing food $\left(\left(\mathrm{P}^{+} / \mathrm{L}^{+}\right) 0.05 \%\right.$, $0.1 \%, 0.3 \%$, and $0.5 \%$ ) as described in Materials and Methods. Panel $B$, snapshots of the first $10 \mathrm{~s}$ of the $0.5 \% \mathrm{Ag} \mathrm{NP}$ (vial on left, marked ' $0.5 \%$ ') versus control (vial on right, marked with ' $C$ '). Analysis was completed by using triplicate sets of data with each set using 15 flies. The bars indicate standard deviation. T-test comparison between the $0.1 \%$ AgNP group and control showed a significant difference with $\mathrm{p}=5.1 \times 10-07$. T-test comparisons between control and $0.05 \% \mathrm{Ag} N \mathrm{P}$ were not significantly different.

bristles appeared devoid of melanin and almost appeared to have a metallic sheen (Figure 4). The flies exposed to $0.3 \%$, $0.1 \%$, and $0.05 \% \mathrm{Ag} \mathrm{NP}$ also showed a concentration dependent reduction in pigmentation (Figure 4 and Table 1). Because many of the flies regained pigmentation as they aged beyond 14 days post-eclosion, the reduced pigmentation resulting from exposure to Ag NP at concentrations of $0.05-0.3 \%$ is transient. Notably, pigmentation was only affected if larvae were exposed to Ag NP, regardless of parental exposure. Furthermore, flies reared on dietary zinc nanoparticles (10-100 nm size) in concurrent experiments have never exhibited the de-pigmentation phenotype seen with Ag NP (data not shown). These data suggest that Ag NP exposure during larval development specifically affects pigmentation in adult flies. 
Because the w1 118 strain of flies lack eye pigmentation, the impact of Ag NP exposure on eye color was tested by using the Oregon $\mathrm{R}$ adult flies. Comparing the eye color of control-fed F1 generation males (24 hr old and virgin) to $1.0 \% \mathrm{Ag} \mathrm{NP} \mathrm{F1}$ generation reared males ( $24 \mathrm{hrs}$ old), we observed that the flies from the exposed parental/larval $\left(\mathrm{P}^{+} / \mathrm{L}^{+}\right)$group lacked body pigmentation while the eye color was virtually unaffected (Figure $5 A$ and $B$ ). In this strain, the contrast between the pigmented bristles on control-fed and the unpigmented bristles of the $\mathrm{Ag}$ NP fed flies is more pronounced (Figure 5B, arrows). This result suggests that the observed de-pigmentation due to Ag NP exposure is specifically affecting body or cuticular pigmentation. Taken together, the observations support the hypothesis that the phenotypic characteristic of losing cuticular pigment is exposuretiming specific (larval stages) and Ag NP dependent.

\section{Stage Specific Exposure related to Life Span Reduction}

The Fl generation larvae exposed to high levels of Ag NP $\left(\mathrm{L}^{+}\right)$suffered reduced longevity. Fl generation flies exposed to $1.0 \%-5.0 \%$ Ag NP-containing food during their larval stage only lived at most $2 \pm 0.3$ days post-eclosion without producing progeny, (F2 generation, Table 1). However, F1 generation flies exposed to lower Ag NP concentrations during their larval stage lived longer, 18-20 days, with life spans comparable to the control group, 20 \pm 1 days, and successfully reproduced (F2 generation, Table 1). Only the $1.0-5.0 \%$ exposed flies lived significantly fewer days than control flies (all $p$-values less than 0.0011 , Table 1 legend). The role of Ag NP exposure during the larval stages of Drosophila development became even more clear when the $\mathrm{Fl}$ generation adults arising from non-exposed larvae (L-) were transferred to food vials with various Ag NP concentrations: these flies retained their pigmentation and all of the flies lived as long as control-fed flies in a 20-day exposure time $(80 \pm 10 \%$ flies survived in all groups, $\mathrm{p}<0.05)$. Further, exposure of the parental $(P)$ generation to Ag NP did not significantly affect the life span of the $F 1$ generation adult flies (data not shown). All these observations indicate the significance of exposure timing, especially during the larval stage, on the longevity of adult flies.

\section{Compromised Climbing Behavior Among Drosophila Reared on the Ag NP Diet}

Because pigmentation defects can be linked to developmental abnormalities including reduction in locomotor ability (Walter et al., 1991; Wright et al., 1976; Drapeau et al., 2003; Neckameyer et al., 2001; Suh and Jackson, 2007), we wanted to assess whether Fl generation adult flies arising from larvae reared on a Ag NP diet exhibited decreased locomotor behavior. To accomplish this we used the climbing behavior assay as previously described (Jimenez-Del-Rio et al., 2010; Chaudhuri et al., 2007). Briefly, groups of ten Fl generation, Ag NPreared flies $\left(\mathrm{P}^{+} / \mathrm{L}^{+}\right)$versus control $\mathrm{Fl}$ generation flies $\left(\mathrm{P}^{-} / \mathrm{L}^{-}\right)$were compared for their ability to climb a vial wall approximately $7 \mathrm{~cm}$ in height after being tapped down.

Since flies arising from the food with $1.0 \% \mathrm{Ag}$ NP were few in number, climbing analysis was carried out on $\mathrm{Fl}$ generation flies emerging from an exposure concentration of $0.5 \% \mathrm{Ag}$ NP and lower. The rate of locomotion of the exposed adult flies seemed to be inversely proportional to the Ag NP concentration. Analysis of digital videography confirmed that the rate of negative geotaxis is inversely proportional to the amount of Ag NP in the diet. Comparing the $0.05 \%$ Ag NP group to the $0.5 \%$ group, the observed decrease in the rate of climbing is from $2.25 \mathrm{~cm}$ $\mathrm{s}^{-1}$ to $0.55 \mathrm{~cm} \mathrm{~s}^{-1}$ (Figure 6A). A T-test comparison of the climbing rates of $\mathrm{Fl}$ generation adult flies in the unexposed group versus the exposed group $(0.1 \% \mathrm{Ag} \mathrm{NP})$ suggested that the difference is statistically significant (at a p-value less than 0.05 , Figure $6 \mathrm{~A}$ ). In Figure $6 B$ is an example taken from the video recordings, illustrating that the organisms fed the high Ag NP concentration $(0.5 \% \mathrm{Ag} \mathrm{NP})$, climb at a slower rate than unexposed, control flies at the time marks of $0 \mathrm{~s}, 5 \mathrm{~s}$, and $10 \mathrm{~s}$ post tap-down (Figure 6B, compare $0.5 \%$ Ag NP-fed flies on left to control (C) flies on right). Regardless of the exposure concentration levels, the multiple sets of one-minute videos revealed that once the $\mathrm{Ag}$ NP-fed flies reached the apex of their climb, their movement essentially ceased (see supplementary video footage). In general, the higher the Ag NP exposure level, the shorter the distance traversed in the allotted 60 second time period. For example, the majority of flies emerging from the larvae exposed to $0.3 \%$ and $0.5 \%$ Ag NP diets were observed to climb for $10-20$ seconds, halt at the $0-4 \mathrm{~cm}$ mark, and appear to tremble in place for the remainder of time. In contrast, the majority of flies resulting from developmental doses of $0.1 \%$ and $0.05 \% \mathrm{Ag} \mathrm{NP}$ climbed to the apex of $\sim 6.5 \mathrm{~cm}$ at rates approximating control rates (within 20 s or 10 s respectively), but again exhibited reduced lateral movement and jumping activity compared to control fed flies. Taken together, the observations suggest that exposure to dietary Ag NP during larval development appears to reduce climbing behavior in emergent Drosophila melanogaster adult flies.

\section{Discussion}

In this study, we have exposed Drosophila melanogaster to dietary silver nanopowder (Ag NP) at different developmental stages to determine the overall effects on the fly life cycle. The results suggest that exposure during the parental stage ( $P$ generation) does not significantly affect developmental progression of the Fl generation larval progeny (L) and that Ag NP-exposure levels of $0.3 \%$ resulted in an overall survival rate that approximated an LD50. Specifically, Ag NP-exposure during F1 generation larval development appeared to negatively impact larval progression, decreased Fl generation adult longevity, altered Fl generation adult locomotor behavior, and decreased Fl generation adult pigmentation. In humans, exposure to silver has been documented to cause medical conditions such as argyria, an irreversible grayish discoloration of the skin (Payne et al., 1992; Chen and Schluesener, 2008; Kwan et al., 2009). As levels of Ag NP are now detectable in the human environment (Kim et al., 2010; Ahamed et al., 2010; Chen and Schluesener, 2008), it is imperative to investigate the entire spectrum of impacts on health. 
De-pigmentation may be due to induction of a stress response. Previous reports indicate that increased stress levels can effect pigmentation, and may be associated with increased expression of heat shock protein 70 (hsp70) (Denman et al., 2008; Galvan and Alonso-Alvarez, 2009; Glassman, 201 1). Posgai et al showed that $3 \mathrm{rd}$ instar larvae feeding on $50 \mathrm{mg} / \mathrm{ml}$ and 100 $\mathrm{mg} / \mathrm{ml}$ coated Ag NP of $10 \mathrm{~nm}$ in size have increased levels of proteins responding to stress including glutathione $\mathrm{S}$ transferase (GST), superoxide dismutase (SOD) and heat shock protein 70 (hsp70) (Posgai et al., 2009). Exposure to zinc nanoparticles ( $Z n$ NP) also induces a stress response and increases the level of hsp70 in marine organisms (Heng et al., 2010; Wong et al., 2010). However, in the present study Drosophila exposed to $\mathrm{Zn}$ NP in comparable amounts to the Ag NP exposures did not lose pigmentation (data not shown). From these observations and previous findings, the mechanism(s) through which Ag NP exposure is associated with de-pigmentation in fruit flies seems to be exposure agent specific.

The mechanisms through which Ag NP exposure during F1 larval stages may cause de-pigmentation, reduced longevity, and reduced locomotor activity in fruit flies could be multi-factorial. While eye pigmentation involves a myriad of genes performing various functions (Lloyd et al., 1998), pigmentation of the adult cuticle (or body) results predominantly from the defined biochemical synthesis of melanins via the melanization/dopamanine pathway. This pathway has been well described and involves a number of enzymes encoded by genes such as: pale, yellow, Dopa decarboxylase (Ddc), and ebony (Sugumaran, 2009; Sugumaran et al., 1992; Wittkopp and Beldade, 2009; Wittkopp et al., 2003; Tang, 2009; Carroll, 2005; Han et al., 2002). Dopamine metabolism is known to generate reactive oxygen species (ROS) which can affect the nervous system and overall negatively affect longevity (Perez and Hastings, 2004). In a previous report, it was shown that fly strains with short life spans appeared to have increased pigmentation compared to long-lived fly strains and that deeper pigmentation was associated with increased dopamine levels, increased oxygen consumption, and increased locomotor activity which indirectly assesses ROS levels (Vermeulen et al., 2006). In contrast, our study suggested that loss of pigmentation is associated with decreased longevity and decreased locomotor activity. Thus, phenotypes arising from Ag NP exposure during larval development may be due to a combination of factors in both the pigmentation and the stress response pathways. Ag NPs may act through one of the following mechanisms: 1) Ag NPs may decrease dopamine levels directly by targeting dopamine pathway genes, enzymes, or localization of melanins; or 2) Ag NPs may elicit the stress response, increasing hsp70 expression and generating ROS, or 3) a combination of both mechanisms. Future studies on the effect of Ag NPs in whole organisms should focus on delineating relevant pathways related to dopamine synthesis, stress response, or some combination.

\section{Acknowledgements}

The authors thank Dr. Mitch McVey at Tufts University for manuscript critique, Dr. Eric Spana at Duke University for Oregon R flies, Bloomington Stock Center for w1 118 flies, the reviewers for constructive feedback, Dr. Mark Wiesner and Dr. Appla R Badireddy at the Center for Environmental Implications of Nanotechnology at Duke University, Department of Civil and Environmental Engineering for their technical support in nanomaterial characterization.

\section{References}

Ahamed M, MS Alsalhi and MK Siddiqui (2010a) Silver nanoparticle applications and human health. Clin Chim Acta $411(23 / 24)$ : $1841-1848$.

Ahamed M, R Posgai, TJ Gorey, M Nielsen, SM Hussain and JJ Rowe (2010b) Silver nanoparticles induced heat shock protein 70, oxidative stress and apoptosis in Drosophila melanogaster. Toxicol Appl Pharmacol 242 (3): 263-269.

AshaRani PV, G Low Kah Mun, MP Hande and S Valiyaveettil (2009) Cytotoxicity and genotoxicity of silver nanoparticles in human cells. ACS Nano 3 (2): 279-290.

Braydich-Stolle L, S Hussain, JJ Schlager and MC Hofmann (2005) In vitro cytotoxicity of nanoparticles in mammalian germline stem cells. Toxicol Science 88 (2): $412-419$

Carroll SB (2005) Evolution at two levels: on genes and form. PLoS Biol 3 (7): e245.

Chaudhuri A, K Bowling, C Funderburk, H Lawal, A Inamdar, Z Wang and JM O'Donnell (2007) Interaction of genetic and environmental factors in a Drosophila parkinsonism model. J Neurosci 27 (10): 2457-2467.

Chen X, and HJ Schluesener (2008) Nanosilver: a nanoproduct in medical application. Toxicol Lett 176 (1): 1-12.

Choi J. E, S Kim, J H. Ahn, P Youn, JS Kang, K Park, J Yi and DY Ryu (2009) Induction of oxidative stress and apoptosis by silver nanoparticles in the liver of adult zebrafish. Aquat Toxicol $100(2): 151-159$

Denman CJ, J McCracken, V Hariharan, J Klarquist, K OyarbideValencia, JA Guevara-Patino and IC Le Poole (2008) HSP70i accelerates depigmentation in a mouse model of autoimmune vitiligo. J Invest Dermatol 128 (8): 2041-2048.

Drapeau MD, A Radovic, PJ Wittkopp and AD Long (2003) A gene necessary for normal male courtship, yellow, acts downstream of fruitless in the Drosophila melanogaster larval brain. J Neurobiol 55 (1): 53-72.

Erickson BE 2009. Nanosilver Pesticides. In Chemical and Engineering News 87(48): 25-26.

Fernandez A, P Picouet and E Lloret (2010) Reduction of the spoilage-related microflora in absorbent pads by silver nanotechnology during modified atmosphere packaging of beef meat. J Food Prot 73 (12): 2263-2269.

Galvan I and C Alonso-Alvarez (2009) The expression of melanin-based plumage is separately modulated by exogenous oxidative stress and a melanocortin. Proc Biol Sci 276 (1670): 3089-3097.

Glassman SJ (2011) Vitiligo, reactive oxygen species and Tcells. Clin Sci (Lond) 120 (3): 99-120.

Han Q, J Fang, H Ding, JK Johnson, BM Christensen and J Li (2002) Identification of Drosophila melanogaster yellow-f and yellow-f 2 proteins as dopachrome-conversion enzymes. 
Biochem J 368 (Pt 1): 333-40.

Heng BC, X Zhao, S Xiong, KW Ng, FY Boey and JS Loo (2010) Toxicity of zinc oxide $(\mathrm{ZnO})$ nanoparticles on human bronchial epithelial cells (BEAS-2B) is accentuated by oxidative stress. Food Chem Toxicol 48 (6): 1762-6.

Jimenez-Del-Rio M, C Guzman-Martinez and C Velez-Pardo (2010) The effects of polyphenols on survival and locomotor activity in Drosophila melanogaster exposed to iron and paraquat. Neurochem Res 35 (2): 227-38.

Jonas, L, C Bloch, R Zimmermann, V Stadie, GE Gross and SG Schad (2007) Detection of silver sulfide deposits in the skin of patients with argyria after long-term use of silver-containing drugs. Ultrastruct Pathol 31 (6): 379-84.

Kim B, CS Park, M Murayama and MF Hochella (2010) Discovery and characterization of silver sulfide nanoparticles in final sewage sludge products. Environ Sci Technol 44 (19): 7509-14.

Kim, S, JE Choi, J Choi, KH Chung, K Park, J Yi and DY Ryu (2009) Oxidative stress-dependent toxicity of silver nanoparticles in human hepatoma cells. Toxicol In Vitro 23 (6): 1076-84.

Kumari A, SK Yadar and SC Yadav (2010) Biodegradable polymeric nanoparticles based drug delivery systems. Colloids Surf B Biointerfaces 75 (1): 1-18.

Kumari M, A Mukherjee and N Chandrasekaran (2009) Genotoxicity of silver nanoparticles in Allium cepa. Sci Total Environ 407 (19): 5243-5246.

Kwon HB, JH Lee, SH Lee, AY Lee, JS Choi and YS Ahn (2009) A case of argyria following colloidal silver ingestion. Ann Dermatol 21 (3): 308-310.

Larese FF, F D'Agostin, M Crosera, G Adami, N Renzi, M Bovenzi and G Maina (2009) Human skin penetration of silver nanoparticles through intact and damaged skin. Toxicology 255 (1/2): 33-37.

Li T, B Albee, M Alemayehu, R Diaz, L Ingham, S Kamal, M Rodriguez and S Whaley Bishnoi (2010) Comparative toxicity study of $\mathrm{Ag}, \mathrm{Au}$, and $\mathrm{Ag}-\mathrm{Au}$ bimetallic nanoparticles on Daphnia magna. Anal Bioanal Chem 398 (2): 689-700.

Liu X, PY Lee, CM Ho, VC Lui, Y Chen, CM Che, PK Tam and KK Wong (2010) Silver nanoparticles mediate differential responses in keratinocytes and fibroblasts during skin wound healing. Chem Med Chem 5 (3): 468-75.

Lloyd V, M Ramaswami and H Kramer (1998) Not just pretty eyes: Drosophila eye-colour mutations and lysosomal delivery. Trends Cell Biol 8 (7): 257-259.

Neckameyer W, J O'Donnell, Z Huang and W Stark (2001) Dopamine and sensory tissue development in Drosophila melanogaster. J Neurobio 47 (4): 280-294.

Payne CM, C Bladin, AC Colchester, J Bland, R Lapworth and $D$ Lane (1992) Argyria from excessive use of topical silver sulphadiazine. Lancet 340 (8811): 126.

Perez RG and TG Hastings (2004) Could a loss of alpha-synuclein function put dopaminergic neurons at risk? J Neurochem 89 (6): 1318-1324.

Posgai R, M Ahamed, SM Hussain, JJ Rowe and MG Nielsen (2009) Inhalation method for delivery of nanoparticles to the Drosophila respiratory system for toxicity testing. SciTotal Environ 408 (2): 439-443.
Powers CM, N Wrench, IT Ryde, AM Smith, FJ Seidler and TA Slotkin (2010) Silver impairs neurodevelopment: studies in PC1 2 cells. Environ Health Perspect 118 (1): 73-79.

Rand MD (2010) Drosophotoxicology: the growing potential for Drosophila in neurotoxicology. Neurotoxicol Teratol 32 (1): 74-83.

Rubin GM (2000) Drosophila Genome Sequence Completed. In HHMI Research News: http://www.hhmi.org/news/rubin3. html.

Sharma VK, RA Yngard and Y Lin (2009) Silver nanoparticles: green synthesis and their antimicrobial activities. Adv Colloid Interface Science 145 (1/2): 83-96.

Sugumaran M (2009) Complexities of cuticular pigmentation in insects. Pigment Cell Melanoma Res 22 (5): 523-5.

Sugumaran M, L Giglio, H Kundzicz, S Saul and V Semensi (1992) Studies on the enzymes involved in puparial cuticle sclerotization in Drosophila melanogaster. Arch Insect Biochem Physiol 19 (4): 271-283.

Suh J and FR Jackson (2007) Drosophila ebony activity is required in glia for the circadian regulation of locomotor activity. Neuron 55 (3): 435-447.

Tang $\mathrm{H}$ (2009) Regulation and function of the melanization reaction in Drosophila. Fly (Austin) 3 (1): 105-111.

Tang J, L Xiong, G Zhou, S Wang, J Wang, L Liu, J Li, F Yuan, S Lu, Z Wan, L Chou and T Xi (2010) Silver nanoparticles crossing through and distribution in the blood-brain barrier in vitro. J Nanosci Nanotechnol 10 (10): 6313-6317.

Vermeulen CJ, TI Cremers, BH Westerink, L Van De Zande and $\mathrm{R}$ Bijlsma (2006) Changes in dopamine levels and locomotor activity in response to selection on virgin lifespan in Drosophila melanogaster. Mech Ageing Dev 127 (7): 610-617.

Walter MF, BC Black, G Afshar, AY Kermabon, TR Wright and $H$ Biessmann (1991) Temporal and spatial expression of the yellow gene in correlation with cuticle formation and dopa decarboxylase activity in Drosophila development. Dev Biol 147 (1): 32-45.

Wittkopp PJ and P Beldade (2009) Development and evolution of insect pigmentation: genetic mechanisms and the potential consequences of pleiotropy. Semin Cell Dev Biol 20 (1): 65 71 .

Wittkopp PJ, SB Carroll and A Kopp (2003) Evolution in black and white: genetic control of pigment patterns in Drosophila. Trends Genet 19 (9): 495-504.

Wong SW, PT Leung, AB Diurisic and KM Leung (2010) Toxicities of nano zinc oxide to five marine organisms: influences of aggregate size and ion solubility. Anal Bioanal Chem 396 (2): 609-618.

Wright TR, GC Bewley and AF Sherald (1976) The genetics of dopa decarboxylase in Drosophila melanogaster. II. Isolation and characterization of dopa-decarboxylase-deficient mutants and their relationship to the alpha-methyl-dopa-hypersensitive mutants. Genetics 84 (2): 287-310.

Wu Y, Q Zhou, H Li, W Liu, T Wang and G Jiang (2009) Effects of silver nanoparticles on the development and histopathology biomarkers of Japanese medaka (Oryzias latipes) using the partial-life test. Aquat Toxicol 100 (2): 160-167. 